\title{
An energy model for the optimal design of linear continuum structures
}

\author{
J.E. Taylor \\ Department of Aerospace Engineering, University of Michigan, Ann Arbor, Michigan 48109, USA
}

\begin{abstract}
An analytical model is presented for the optimal design of linearly elastic continuum structures. To facilitate the expression of the combined analysis and design problem in general form, a basis is introduced covering a general set of energy invariants. Both internal (strain) energy and the expression of generalized cost are represented conveniently in terms of this basis, and as a result the optimality conditions for the design problem have a particularly simple form. Present developments comprise a reinterpretation and an extension of existing models where the design variable is the material modulus tensor, and where "cost" is represented in a general form. The conventional potential energy statement for linear continuum elastostatics is restated in the form of an isoperimetric problem, as a preliminary step. This interpretation of the mechanics is then incorporated in a max-min formulation applicable for the general design of linear continuum structures. To exemplify its application, the model is interpreted as it would apply for certain materials with particular geometric structure, e.g. crystalline forms. Also problems treated earlier where optimal material properties are predicted for the case where unit cost is proportional to the trace of the modulus tensor are identified as examples within the generalized formulation. The application of a recently developed technique to predict optimal black-white structures, i.e. designs having sharp topological features, is considered in the setting of the present generalized model.
\end{abstract}

\section{Introduction}

The developments reported in this paper lie in the area of formulations for structural optimization where optimal material properties may be predicted at once along with other attributes of design such as material distribution, shape, and layout or topology. For the linearly elastic continuum structure, the model for this design problem stated in its primitive form has the unrestricted modulus tensor appear in the role of "design variable". In past treatments of the problem, the constraint on total resource or cost is expressed in explicit, assumed form, i.e. a form where the argument in the constraint is designated as one or another among specific invariant measures associated with the material modulus tensor. In the present formulation this earlier model is extended by having the global cost constraint represented in a general form. The expression for generalized cost is constructed in a way to accommodate all possible nonnegative, invariant measures of the material that are linear in the modulus tensor. Introduction of an energy basis in the analytical modelling leads to a particularly simple form for the statement of the contin- uum structural design problem, and for the expression of the associated governing equations. Both the fundamental elastostatics analysis problem and its extension to predict the optimal continuum structure are expressed in terms of the basis in the process. An interpretation is given to identify earlier models as examples imbedded within the generalized cost formulation. Other applications to solve for black/white or "topology" design, and for the prediction of optimal continua within restrictions on local attributes of the material tensor, are described as well.

A treatment for the optimal design of the material modulus tensor field was presented first by Bends $\varnothing$ e et al. (1994); the paper includes a statement of the problem formulation, its analytical interpretation, and computational results for an example problem. The design problem treated in that paper and in subsequent elaborations to this approach for the design of optimal continuum structures (see e.g. Bendsøe et al. 1995,1996 ) is the basic (and overworked!) problem having the objective to minimize compliance. The approach to design optimization having pointwise material properties as the design variables is notably distinct from the more familiar, precedent characterizations, where the models make use of a homogenization interpretation (or an approximation thereof) for a locally two-phase composite representation of structural material. The survey article by Rozvany et al. (1995), and the proceedings edited by Bendsøe and Mota Soares (1993) are cited as useful recent publications where activity in the latter subject area is surveyed and documented. The entire subject of design optimization to predict local properties along with shape and material distribution is surveyed broadly and very effectively in the treatise by Bendsøe (1995). Very recent developments applicable to 3D topology design are reported by Olhoff et al. (1997). Useful contrasts may be drawn between continuum modelling for arbitrary materials, as represented in studies with the modulus tensor as design variable, and the treatment for optimal design of anisotropic structures; extensive work by Pedersen in the latter area is summarized in the paper by Pedersen (1993). The factor of "arbitrariness of directional properties" also arises within a generalized study of a class of structures in the inspired work of Rozvany (1976) on the optimal design of flexural systems.

A formulation for optimal continuum structural design having the cost constraint expressed in generalized form is presented in the work of Taylor and Washabaugh (1995a,b, 1997). The selfsame technique, namely the introduction of a set of unit energies suitable as a basis for the expression 
in general of unit cost, is employed to represent cost in the formulation of this paper. This form is applicable for linear continuum structures modelled according to linear deformation kinematics, i.e. for linear elastostatics. The element of formulation for optimal design introduced here which has system strain energy expressed in terms of the same basis apparently is original.

The development of the paper is presented in four parts. First, to facilitate the eventual expression of the general design problem in compact and convenient form, the conventional "minimum potential energy" statement for linear elastostatics analysis is cast in the form of an isoperimetric problem. The ultimate purpose of the presentation, namely to state the general coupled analysis and design problem in a substantially simplified and uniform form, calls for establishment of a "basis of invariants" suitable for the expression of both energy and cost invariants for the general problem, and this is done next. An interpretation of the measure of total strain energy in terms of this basis is introduced, and this provides for a restatement of the elastostatics problem in final form. Finally, the general combined analysis and optimal design problem is stated using the basis, and an interpretation is given for the associated set of necessary conditions. The system is applied to show how an example of the material design problem treated in earlier studies, namely those where "unit cost" was taken to be proportional to the trace of the modulus tensor, appears according to interpretation under the present problem formulation. Also, a demonstration is given to show how optimal continuum forms having particular geometric features, namely those associated with groups of crystalline material structure, may be generated according to the particular form designated for the argument in the generalized cost constraint. Possible application in the setting of the generalized formulation for a technique (Guedes and Taylor 1997) for prediction of optimal black/white design for continuum structures is discussed as well.

\section{Elastostatics restated in isoperimetric form}

We refer as a starting point to the conventional "minimum potential energy" statement for linear elastostatics of continuum structures. The structure is taken to be strongly stable, i.e. the material tensor, otherwise arbitrary within limits of the continuum model, is assumed to be positive definite over the entire domain of the structure (the requirement is to be reconsidered in the later section on the design problem). For structure occupying region $\Omega$ with boundary $\Gamma$, subject to body force field $f$ and boundary forces $t$, the equilibrium state is identified with the necessary conditions for a minimum of potential energy. The problem is stated symbolically as

$$
\begin{aligned}
& \min _{v \in K}\left\{\Pi=\int_{\Omega}\left[\frac{1}{2} E_{i j k \ell} \varepsilon_{i j}(v) \varepsilon_{k \ell}(v)-f_{i} v_{i}\right] \mathrm{d} V-\right. \\
& \left.\int_{\Gamma_{t}} t_{i} v_{i} \mathrm{~d} S\right\}
\end{aligned}
$$

where $\varepsilon_{i j}(v)$ symbolizes strain linear in admissible displacement $v_{i}$, the material modulus is represented by $E, \Gamma_{t}$ identifies the portion of boundary $\Gamma$ subject to boundary traction, $K$ identifies the set of kinematically admissible displacements, defined as usual for the problem, and all other elements of the problem statement are to be interpreted according to routine convention (the requirement of zero displacement on $\Gamma-\Gamma_{t}$ is assumed for simplicity). For any properly cast version of the problem at hand, the potential energy is convex in displacement, the potential energy is minimized at the solution state, and the system is stable. In the treatment described below for the design problem, the present assumption of uniformly stable material is not invoked and, in that case, the consideration of structural stability is approached differently. The point is addressed in the section on design.

For the next step, the relationship of the following isoperimetric problem to the given potential energy problem statement is to be established. The isoperimetric problem reflects minimization of the strain energy within an isoperimetric constraint on the measure of compliance [this interpretation is applied e.g. by Taylor (1997), and by Achtziger et al. (1997)], i.e.

$\min _{v \in K}\left\{\int_{\Omega}\left[\frac{1}{2} E_{i j k \ell} \varepsilon_{i j}(v) \varepsilon_{k \ell}(v)\right] \mathrm{d} V\right\}$,

subject to

$\underline{W}-\left\{\int_{\Omega} f_{i} v_{i} \mathrm{~d} V+\int_{\Gamma_{t}} t_{i} v_{i} \mathrm{~d} S\right\} \leq 0$.

Data for this problem includes the lower bound $W>0$, as well as the loads and material tensor data of problem [P1]. The relationship between these two problem statements is established on the basis of a comparison of the conditions reflecting stationarity w.r.t. displacement $v_{i}$ for each, and on the demonstration that there exists a value, say $\underline{W}_{1}$, such that the solutions to them are identical. Stationarity in [P1] requires satisfaction of the equilibrium equation and static boundary condition, i.e.

$$
\begin{aligned}
& \left(E_{i j k \ell} \ddot{u}_{i, j}\right)_{, k}+f_{\ell}=0, \quad x \in \Omega, \\
& \left(E_{i j k \ell} \ddot{u}_{i, j}\right) n_{k}-t_{\ell}=0, \quad x \in \Gamma_{t} .
\end{aligned}
$$

Symbols $u$ with over-dots signify "solution function". Similarly, stationarity in [P2] requires

$$
\begin{aligned}
& \left(E_{i j k \ell^{u_{i, j}}}\right)_{, k}+\dot{\Lambda} f_{\ell}=0, \quad x \in \Omega, \\
& \left(E_{i j k \ell} \dot{u}_{i, j}\right) n_{k}-\dot{\Lambda} t_{\ell}=0, \quad x \in \Gamma_{t},
\end{aligned}
$$

and additionally

$\dot{\Lambda}\left[\underline{W}-\left(\int_{\Omega} f_{i} \dot{u}_{i} \mathrm{~d} V+\int_{\Gamma_{t}} t_{i} \dot{u}_{i} \mathrm{~d} S\right)\right]=0, \quad \dot{\Lambda} \geq 0$, 
where $\dot{\Lambda}$ symbolizes the multiplier associated with the isoperimetric constraint. Note that this constraint excludes $\dot{u} \equiv$ 0 , and accordingly from (2) and (3) $\dot{\Lambda}>0$ and $[\underline{W}-$ $\left.\left(\int_{\Omega} f_{i} \dot{u}_{i} \mathrm{~d} V+\int_{\Gamma} t_{i} \dot{u}_{i} \mathrm{~d} S\right)\right]=0$. The latter equation provides for evaluation of multiplier $\dot{A}$, i.e. with the substitution $\dot{u}_{i}=A \ddot{u}_{i}$ [the relation follows from similarity of the linear boundary-value-problem statements (1) and (2)] one finds

$$
\dot{\Lambda}=\frac{\underline{W}}{\left(\int_{\Omega} f_{i} \ddot{u}_{i} \mathrm{~d} V+\int_{\Gamma_{t}} t_{i} \ddot{u}_{i} \mathrm{~d} S\right)} .
$$

Problems [P1] and [P2] are equivalent for $\dot{\Lambda}=1 \rightarrow \dot{u}=\ddot{u}$, and so from (4) the data value $\underline{W}_{1}$ for equivalence is given by

$\underline{W}_{1}=\left(\int_{\Omega} f_{i} \ddot{u}_{i} \mathrm{~d} V+\int_{\Gamma_{t}} t_{i} \ddot{u}_{i} \mathrm{~d} S\right)$.

This completes a confirmation of the representation for elastostatics problems in the form of isoperimetric problem [P2] (literally that problem [P1] is imbedded within [P2]). However, one additional step is required in order to render the variational statement for elastostatics into the form appropriate for subsequent purposes. This modified form, which is entirely equivalent to [P2], has strain and displacement appear as variationally independent quantities, within a constraint to enforce (linear) strain-displacement relations [this form of representation of deformation kinematics within variational formulations is exercised in various other settings; see e.g. Washizu (1982)]. The modified problem is stated symbolically as

$\min _{\varepsilon, v \in K}\left\{\int_{\Omega}\left(\frac{1}{2} E_{i j k \ell} \varepsilon_{i j} \varepsilon_{k \ell}\right) \mathrm{d} V\right\}$

subject to

$\underline{W}-\left\{\int_{\Omega} f_{i} v_{i} \mathrm{~d} V+\int_{\Gamma_{t}} t_{i} v_{i} \mathrm{~d} S\right\} \leq 0$,

$\frac{1}{2}\left(v_{i, j}+v_{j, i}\right)-\varepsilon_{i j}=0, \quad x \in \Omega$.

The character of the "min problem" remains unaltered through this transformation, and the entire discussion given above for the interpretation of [P2] applies equally well to this slightly modified form of the problem.

\section{Basis invariants for the coupled problem}

The object in this section is to establish a basis of invariants suitable for the expression in general of both strain energy and cost, for arbitrary linear material and in the setting of linear elastostatics. The larger subject dealing with invariants of tensors in general, e.g. the material modulus tensor, is not addressed here, nor is it necessary for the purposes of this paper [relatively contemporary treatments of invariants, bases, etc. of general tensors are presented e.g. by Zheng (1994) and Jemiolo and Telega (1997)]. Rather, the aim here is to identify the simplest, self-consistent basis suitable for expression of the combined analysis and design problem, and it appears that this is accomplished with a basis defined in terms of a set of energies for the problem. Suitability of this interpretation is reckoned simply by the properties that both the general material tensor for linear elasticity, and the measure of strain energy for the response in elastostatics as well, can be represented uniquely in terms of the same basis. As noted earlier, a precedent for the approach for the expression of cost used here is described in earlier treatments for generalized cost (Taylor and Washabaugh 1995a,b, 1997). Again, the current presentation differs somewhat in detail from the earlier ones, and it is distinct in particular for its interpretation of the elastostatics (analysis) part of the problem using the energy basis.

The calculations to establish the "energy basis" are described here in algebraic form applicable to the $2 \mathrm{D}$ or $3 \mathrm{D}$ continuum. As a first step, arbitrary strain field, say $\varepsilon_{i j}$, is represented via the construction

$\varepsilon_{i j}(x)=\sum_{\beta=1}^{N} c_{\beta}(x) \eta_{i j}^{\beta}$,

where the $\eta_{i j}^{\beta}$ comprise a set of $N$ linearly independent constant reference (strain) values. The number $N$ corresponds to the number of independent components in the symmetric two-tensor. from:

For physical dimension $D$, this number may be evaluated

$N=D^{2}-\sum_{k=1}^{D-1}(D-k)$.

The designation of reference strains $\eta_{i j}^{\beta}$ is arbitrary within the stated requirements (example calculations using a specific choice for the reference strains are given in Appendix I). Interpretation (6), which has spatial dependence of strain represented through the coefficients $c_{\beta}$, is on its own of no consequence in the modelling of elastostatics. In the present development, however, this device provides directly for the construction of the energy basis, which is to be described next.

A specific basis is constructed for a given material, represented for the constitutively linear problem by arbitrary modulus tensor $E_{i j k \ell}(x)$, and the designated set of reference strains. The first $N$ elements of the basis, symbolized by $I_{\gamma}$, are defined via the simple quadratic expression

$I_{\gamma}(x):=E_{i j k \ell}(x) \eta_{i j}^{\gamma} \eta_{k \ell}^{\gamma}, \quad \gamma=1,2, \ldots, N$

(no sum on $\gamma$ ).

Recall that $N$ is the count of independent components of the symmetric two-tensor. The $I_{\gamma}$ evaluated according to (8) are simply twice the measures of unit strain energy of the 
individual components of reference strain. The remaining elements of the basis are comprised of unit strain energies as well, where each element is identified with one among all possible independent linear combinations of the reference strains. These combinations, symbolized by $\zeta_{i j}^{\mu}$, are formed according to

$\zeta_{i j}^{\mu}=\eta_{i j}^{\alpha}+\eta_{i j}^{\beta}, \quad \alpha \neq \beta, \quad \mu=1,2, \ldots, C$,

where $C$ counts the number of independent combinations of two unlike components within a group of $N$ components. The count $C$ may be calculated from

$C={ }_{N} C_{2}=\frac{N(N-1)}{2 !}$

Also, in the 2D case, the strains $\zeta_{i j}^{\mu}$ indicated in (9) may be evaluated directly from the expression $\left(C_{r s t}\right.$ represents the permutation symbol)

$\zeta_{i j}^{\mu}=-\frac{1}{2} C_{\mu \alpha \beta} C_{\alpha \mu \beta}\left(\eta_{i j}^{\alpha}+\eta_{i j}^{\beta}\right), \quad \alpha, \beta=1,2, \ldots, N$,

$\mu=1,2, \ldots, C, \quad$ (no sum on $\mu$ ).

Thus the additional elements, say $\hat{I}_{\gamma}$, to complete the basis of invariants are the unit energies associated with strains $\zeta_{i j}^{\mu}$ of (9) and the same material $E$, i.e.

$\hat{I}_{\mu}(x):=E_{i j k \ell}(x) \zeta_{i j}^{\mu} \zeta_{k \ell}^{\mu}, \quad \mu=1,2, \ldots, C$,

(no sum on $\mu$ ).

Unit energies (12) combined with those of (8) encompass all possible independent inner products (triples) associated with the given material modulus field $E_{i j k \ell}(x)$ and quadratic in the designated set of reference strains. The complete basis is given by the collection of sets $I_{\gamma}$ and $\hat{I}_{\gamma}$, i.e. with its elements symbolized by $B_{\Phi}(x)$ the basis is described by

$\left\{B_{\Phi}\right\}=\left\{I_{\gamma}\right\}+\left\{\hat{I}_{\mu}\right\}=\left\{I_{1}, I_{2}, \ldots, I_{N} ; \hat{I}_{1}, \hat{I}_{2}, \ldots, \hat{I}_{C}\right\}$.

The total number $N+C$ of elements $B_{\Phi}$ is six in 2D and twenty-one in $3 \mathrm{D}$, just the number of independent components of the modulus tensor for the classical continuum. For a given set of reference strains, relations (13) amount to an expression of $B(E)$. The expression is unique; the evaluation of transformation $B(E)$ and its inverse $E(B)$ is described in Appendix I for a particular numerical choice of the reference strains.

As was suggested earlier, it becomes possible through the introduction of the energy basis to express the optimal design problem in a particularly convenient form. According to the developments just described, in this problem for the design of the optimal material field the basis itself may be taken to comprise the "design variable". Also, while local representation of the material modulus tensor may be expressed effectively with reference to its eigenproperties (see e.g. Lipton 1993; Diaz et al. 1995), the interpretation in terms of the present basis, identified with a fixed reference frame, is more convenient in the treatment of "design within generalized cost"

Before turning to the full description of this design problem, a brief consideration is given next to reinterpretation of the elastostatics alone making use of the energy basis.

\section{Elastostatics restated in terms of the basis}

Restatement of problem [P3] for the general elastostatics response of systems with arbitrary material follows simply from interpretations of strain and then strain energy in terms of the basis strains and invariant basis defined above. Thus with the substitution of response strains stated in terms of the set of reference strains via (7) into the expression for unit strain energy, interpretation of the latter in terms of the "energy basis" (13) follows by a simple calculation, i.e.

$$
\begin{aligned}
& \frac{1}{2} E_{i j k \ell} \varepsilon_{i j} \varepsilon_{k \ell}=\frac{1}{2} E_{i j k \ell}\left(\sum_{\alpha=1}^{N} c_{\alpha} \eta_{i j}^{\alpha}\right)\left(\sum_{\beta=1}^{N} c_{\beta} \eta_{k \ell}^{\beta}\right)= \\
& \ldots:=\sum_{\delta=1}^{N+C} e_{\delta} B_{\delta} .
\end{aligned}
$$

Coefficients $e_{\gamma}(x)$, defined implicitly in the last step of this continued equation, are quadratic in $c_{\gamma}(x)$. (With judicious choice of the basis strains, the expressions for $e_{\gamma}$ have simple form, e.g. as realized with the examples described in Appendix 1.) With the substitutions for response strain via (7) and strain energy according to (14), version [P3] of the elastostatics problem is transformed to

$\min _{c_{\alpha} ; u_{k}}\left\{\int_{\Omega} \sum_{\delta=1}^{N+C} e_{\delta}\left(c_{\alpha}\right) B_{\delta} d V\right\}$,

subject to

$\underline{W}-\left\{\int_{\Omega} f_{i} u_{i} \mathrm{~d} V+\int_{\Gamma_{t}} t_{i} u_{i} \mathrm{~d} S\right\} \leq 0$,

$\frac{1}{2}\left(u_{i, j}+u_{j, i}\right)-\sum_{\gamma} c_{\gamma} \eta_{i j}^{\gamma}=0, \quad x \in \Omega$.

Constants $\underline{W}$, and $\eta_{i j}^{\gamma}$ and fields $f_{k}(x), t_{k}(x)$, and $B_{\delta}(x)$ comprise the data for this form of the problem. As noted earlier, the isoperimetric constraint excludes the possibility $u_{k} \equiv 0$, and the objective in [P4], which measures total strain energy, is definitely positive. The equilibrium boundary value problem statement is identified (still) with stationarity conditions for this version of the problem, where now the state variables are $c_{\alpha}(x)$ and $u_{k}(x)$ (note that the prior requirement $E_{i j k \ell}>0$ applies here as before, i.e. the formulation is intended for structures composed of uniformly stable materials). With the introduction of $q_{i j}$ as the multiplier associated with the kinematic constraint of [P4], the equilibrium system is stated

$$
\begin{aligned}
& \sum_{\delta=1}^{N+C} \frac{\partial e_{\delta}}{\partial c_{\alpha}} B_{\delta}-q_{i j} \eta_{i j}^{\alpha}=0, \quad x \in \Omega, \quad \alpha=1,2, \ldots, N \\
& q_{i j, j}+\Lambda f_{i}=0, \quad x \in \Omega, \quad i=1,2,3 \\
& q_{i j} n_{j}+\Lambda t_{i}=0, \quad x \in \Gamma_{t}, \quad i=1,2,3
\end{aligned}
$$


with $q_{i j} n_{j}=0$ on the remainder of the boundary where displacement is not prescribed. Clearly symbol $q_{i j}$ may be identified as the measure of stress. Accordingly, the first term of $(15)$ is seen to represent the component of total energy associated with the $\alpha$-th element among prescribed reference strains $\eta_{i j}^{\gamma}$; the equation can be recognized in this way as an implicit expression of the constitutive relations. Also from (15) $q_{i j} \neq 0$ almost everywhere, and so the constraint on deformation kinematics is enforced; it follows as well from (16) that $\Lambda \neq 0$.

While there is no apparent advantage per se to version [P4] over more familiar variational forms for elastostatics analysis, recall that our purpose was to derive an expression that should be particularly convenient for a unified statement of the optimal design problem for elastostatic structural design. It will become apparent with the developments of the next section how this formulation [P4] is useful toward that purpose.

\section{The combined analysis and design problem}

As indicated in the Introduction, in existing treatments for optimization of continuum structures having the form where design is represented by the free material modulus tensor (e.g. Bendsøe et al. 1994, 1995), the isoperimetric or "cost" constraint is expressed in terms of one choice or another from among select invariants of the modulus tensor. This was done as well in the extension of such formulations to establish a procedure for the prediction of "sharp image" (topology design) versions of optimal structures (Guedes and Taylor 1997). So far as one may be concerned with the significance of how the measure of cost is designated, such formulations themselves amount to examples and clearly there is purpose to consider more general statements of the cost constraints. Where the measure of cost is to be linear in (elements of) the modulus tensor, Washabaugh and Taylor (1995a,b, 1997) describe means to express the cost constraint in general form. Generality here follows from the fact that the expression provides a unique measure for any such given material, and that it is invertible. The "basis of invariants" described in the latter papers has precisely the form of the basis described above, and so for present purposes we simply assert that the generalized cost is expressed as the integral over the structure of an arbitrary linear function within the set of functions described in terms of this basis. Thus for a specified bound $R$ on resource, the cost constraint is stated

$\int_{\Omega}\left[\sum_{\gamma} b_{\gamma}(x) B_{\gamma}(x)\right] \mathrm{d} V-R \leq 0, \quad b_{\gamma}>0 \quad \forall \gamma$.

Variation of cost over the structure is reflected in the coefficients $b_{\gamma}(x)>0$ of the linear expression, and as indicated these coefficients are limited only to be positive-valued in position coordinates, i.e. total cost per unit volume lies in the "first quadrant". The coefficients are specified for the optimal design problem. They may be interpreted to represent componentwise relative unit cost of the material. Generality of this form of expression for cost derives from completeness of the basis set $B_{\gamma}$.
For the present statement of the design problem where the elastostatics and the cost constraint are represented via [P4] and (17), the basis elements $B_{\gamma}$ themselves have the role of "design variables". Thus with upper and lower bounds $\bar{B}_{\gamma}$ and $\underline{B}_{\gamma}$ on the local value of $B_{\gamma}$, the design problem is stated symbolically in the form of the maxmin problem:

$\max _{B_{\gamma}}$

subject to

$$
\begin{aligned}
& \int_{\Omega}\left(\sum_{\gamma} b_{\gamma} B_{\gamma}\right) \mathrm{d} V-R \leq 0, \quad 0<\underline{B}_{\gamma} \leq B_{\gamma} \leq \bar{B}_{\gamma}, \\
& \min _{c_{\alpha}(x) ; u_{k}(x)}\left\{\iint_{\Omega} \sum_{\gamma} e_{\gamma}\left(c_{\alpha}\right) B_{\gamma} \mathrm{d} V\right\},
\end{aligned}
$$

subject to

$$
\begin{aligned}
& \underline{W}-\left(\int_{\Omega} f_{i} u_{i} \mathrm{~d} V+\int_{\Gamma_{t}} t_{i} u_{i} \mathrm{~d} S\right) \leq 0, \\
& \frac{1}{2}\left(u_{i, j}+u_{j, i}\right)-\sum_{\gamma} c_{\gamma} \eta_{i j}^{\gamma}=0 .
\end{aligned}
$$

The "max" of total strain energy w.r.t. the basis energies does in fact correspond to the intended objective to minimize compliance. This correspondence is confirmed in Appendix II. Noting that design variable $B_{\gamma}$ appears only in the argument of the integral within " $\mathrm{min}$ " of maxmin problem [D1], the "max" or design part of the problem can be interpreted independently [the step is argued by Bendsøe et al. (1994) in terms of an interchange of max and min] as

$\max _{B_{\gamma}}\left\{\int_{\Omega} \sum_{\gamma} e_{\gamma} B_{\gamma} \mathrm{d} V\right\}$

subject to

$\int_{\Omega}\left(\sum_{\gamma} b_{\gamma} B_{\gamma}\right) d V-R \leq 0, \quad 0<\underline{B}_{\gamma} \leq B_{\gamma} \leq \bar{B}_{\gamma}$.

Maximization in [D2] relates in fact to local control of the $B_{\gamma}$. Introducing $K, \bar{\kappa}_{\gamma}$, and $\underline{\kappa}_{\gamma}$ as multipliers on the isoperimetric constraint and the local upper and lower bounds respectively, stationarity w.r.t. $B_{y}$ requires (satisfaction of the "optimality conditions")

$$
\left.\begin{array}{l}
-e_{\gamma}+\bar{\kappa}_{\gamma}-\underline{\kappa}_{\gamma}+b_{\gamma} K=0, \\
\bar{\kappa}_{\gamma}\left(B_{\gamma}-\bar{B}_{\gamma}\right)=0, \quad \bar{\kappa}_{\gamma} \geq 0, \\
\underline{\kappa}_{\gamma}\left(\underline{B}_{\gamma}-B_{\gamma}\right)=0, \quad \geq 0,
\end{array}\right\} x \in \Omega, \gamma \in G_{\gamma},
$$


where $G_{\gamma}$. identifies the set of all values gamma. It is assumed that the data value $R$ lies in the range such that at least one among the design variables $B_{\gamma}$ satisfies $\underline{B}_{\gamma}<B_{\gamma}<\bar{B}_{\gamma}$, i.e. lies off the local constraints at least somewhere in $\Omega$. Identifying such intervals and the set of associated indices respectively by $\Omega_{D \gamma}$ and $G_{D \gamma}$, from (18)

$e_{\gamma}=b_{\gamma} K, \quad x \in \Omega_{D \gamma}, \quad \gamma \in G_{D \gamma}$.

Since $b_{\gamma}>0$ for all gamma and over the entire structure, if $K=0$ then according to $(20) e_{\gamma}=0$ for all $\gamma \in G_{D \gamma}$. This implies in turn that the optimal structure includes components $B_{\gamma}>\underline{B}_{\gamma}$ with positive cost and zero contribution to the objective of "max", and of course this is a contradiction. Accordingly, the solution value of $K$ satisfies $K>0$, and so $e_{\gamma}>0 \forall x \in \Omega_{D_{\gamma}} ; \gamma \in G_{D_{\gamma}}$, and also from (19)

$$
\int_{\Omega} \sum_{\gamma} b_{\gamma} B_{\gamma} \mathrm{d} V-R=0 .
$$

To consider further the issues related to stability, note that the deduction $e_{\gamma}>0$ applies componentwise and locally in $\Omega$. Thus, for example, the system admits the possibility of a solution where there is no interval within the structure having

$e_{\gamma}>0$, for all $\gamma \in G_{\gamma}$.

Stated differently, there is no assurance that the solution to the set of necessary conditions excludes a negative contribution to strain energy for one or more components among $\gamma \in G_{\gamma}$. Nonetheless, considering the solution to problem [D1], i.e. the putative optimal design, the total strain energy associated with it is definitely positive; this is substantiated within material presented in Appendix II. For the standard linear continuum elastostatics problem, this assures that the loaded structure is (globally) stable. In other words, the solution to optimal material design problem [D1] (where it exists) identifies a stable structure independent of explicit local requirement on the material properties. Accordingly, it would be redundant in this setting to impose a local constraint of the form $E>0$, i.e. to enforce the pointwise condition for a strongly stable material, out of concern about (global) stability. Note that in the present formulation for the design problem the local constraint to ensure that $B_{\gamma}>0 ; x \in \Omega$; $\gamma \in G_{\gamma}$ is essential, and this constraint implies through the relation $B(E)$ a restriction on the modulus tensor itself.

To summarize explicitly the remaining possibilities within (18), note from the complementarity conditions there that the two local constraints on design cannot be active simultaneously, i.e. $\underline{\kappa}_{\gamma} \bar{\kappa}_{\gamma}=0$ and so for $x \notin \Omega_{D}$,

either $\bar{\kappa}_{\gamma}>0 \rightarrow B_{\gamma}=\bar{B}_{\gamma}$ and $e_{\gamma}=K b_{\gamma}+\bar{\kappa}_{\gamma}$,

say for $x \in \bar{\Omega}_{\gamma}$,

or $\underline{\kappa}_{\gamma}>0 \rightarrow B_{\gamma}=\underline{B}_{\gamma}$ and $e_{\gamma}=K b_{\gamma}-\underline{\kappa}_{\gamma}$,

say for $x \in \underline{\Omega}_{\gamma}$, or $\bar{\kappa}_{\gamma}=0$ and $B_{\gamma}=\bar{B}_{\gamma}$ and $e_{\gamma}=K b_{\gamma}+\bar{\kappa}_{\gamma}$

say for $x \in \bar{\Omega}_{\gamma 0}$,

or $\underline{\kappa}_{\gamma}=0$ and $B_{\gamma}=\underline{B}_{\gamma}$ and $e_{\gamma}=K b_{\gamma}-\underline{\kappa}_{\gamma}$,

say for $x \in \underline{\Omega}_{\gamma 0}$.

The last two lines represent the conditions at intersections between $\Omega_{\gamma D}$ and $\underline{\Omega}_{\gamma}$, and between $\Omega_{\gamma D}$ and $\bar{\Omega}_{\gamma}$, respectively. This interpretation of the original optimality condition (9) is exhaustive, and we have the closure

$\Omega_{\gamma D} \cup \bar{\Omega}_{\gamma} \cup \underline{\Omega}_{\gamma} \cup \bar{\Omega}_{\gamma 0} \cup \underline{\Omega}_{\gamma 0}=\Omega$.

Since problem [D1] is a maxmin problem, the system comprised of the necessary conditions plus the original constraints is in fact sufficient to identify the optimal design and associated equilibrium response state. In other words, the system serves to identify a unique solution to the general design problem. Again, a confirmation of the correspondence between [D1] and the intended objective of "minimum compliance" is presented in Appendix II.

Before quitting this section on modelling to consider example applications, a technique for the prediction of black/white design mentioned earlier is discussed to indicate its interpretation in the setting of the generalized cost problem. Reference is made to the particular approach presented by Guedes and Taylor $(1997 a, b)$ for the case where the isoperimetric (cost) constraint is expressed in terms of the trace measure of the modulus tensor. As described in the cited paper, it is necessary in this approach to modify the argument of the global cost constraint by the addition of a (prescribed) weighting function or relative unit cost coeffcient. This weighting function corresponds precisely in terms of its significance to the coefficients $b_{k}(x)$ in the global cost constraint for the present generalized cost formulation, and so the same method is directly applicable in the setting of the "general cost" formulation. With some elaboration in the computational procedure, the system provides the possibility of generating one or more black/white designs, each one associated with an element of the basis set $B_{k}(x)$ of design functions. This point is discussed further in the Conclusions.

\section{Example applications}

Formulation [D1] for the design of continuum structures covers the generally inhomogeneous structure having arbitrary local constitution of material, the former following from the representation of both state, in terms of $c_{\gamma}(x)$, and design unit cost $b_{\gamma}(x)$ as relatively unrestricted functions. The model is general as well in the sense that a full range of optimal materials may be predicted out of its solution, corresponding to the breadth of coverage in stating "cost" in the cost constraint. This is in contrast to the problems described in earlier treatments for the design of the material tensor, where in each case a specific form of cost is assumed and the resulting solution identifies a specific constitution of the material. Acknowledging that - relative to the range of 
possibilities for its application -- exemplification via a limited set of specific problems comprises at best a sharply limited demonstration of the model, the following kinds of problems are to be described.

- An example from among treatments presented in the literature on design of continuum structures, namely the optimal design of a Lamé cylinder where local cost is taken to be proportional to the trace of the modulus tensor, is interpreted within the present formulation. The solution itself is taken from existing results (Guedes and Taylor 1997b) for this problem. A recently developed technique for the prediction of optimal black/white design is interpreted in the setting of the present generalized formulation, also using this example as a reference case. A graphical presentation of this solution for the "sharp image" optimal design is given as well.

- The general formulation is to be interpreted with the purpose in mind to predict optimal design where the local properties are identified in a system for classification with one or another group, according to its symmetry properties. Here we follow the classification scheme for crystal structures as given by Love (1926), and treat the monoclinic and rhombic groups as examples. The correlation between optimal design having material symmetries belonging to each of the groups and the respective argument of the cost constraint in [D1] is addressed.

\subsection{For cost proportional to the trace of the modulus tensor}

The original report on a model for optimal design where the design variable is the material modulus (Bendsøe et al. 1994) treats the case where total cost is proportional to the integral over the structure of the trace of the modulus tensor. The solution in this case and for minimum compliance design of the single purpose structure is verified (in analytical form) to be a zero-shear-stiffness, orthotropic material aligned with the principal directions of the strain tensor. This result may be viewed as an example of how a unique material structure derives from the designation of a specific measure of local cost, namely the trace $E_{i j i j}$ of the modulus tensor. An interpretation of the present formulation [D1] to correspond to the original study is obtained simply by replacing the "total cost" constraint there by

$\int_{\Omega} E_{i j i j} \mathrm{~d} V-R \leq 0$.

The result is obtained for problems in 2D, for example, with chosen constant values $b_{1}=b_{2}=b_{3}=1 / 2 ; b_{4}=b_{5}=b_{6}=0$ for the unit cost coefficients in [D1]. Note, however, that the designation of zero values here is inconsistent with the requirement $b_{\gamma}(x)>0, \forall \gamma$ of (17). An appropriate interpretation of the general formulation is obtained for this example by assuming the values to be small rather than zero. (In the original study, the existence of the solution is argued for the form of the cost constraint assumed there, and this amounts to a confirmation of that problem as a limit case with the small values approaching zero within the general model.) The distinction may be important in the respect that while the general formulation is valid for all problems that can be simulated within the cost constraint of [D1], any specific form of cost outside those admissible in the general formulation would require independent confirmation. For the purposes of this demonstration, attention is restricted to examples covered within the present formulation. On another point, one may note that the presence of unit cost coefficients within the general formulation makes it possible to study how the character of optimal local material properties varies with continuous variation of these factors. Studies of this kind have not yet been performed.

Computational results are shown in Fig. 1a for the example problem taken from the paper by Guedes and Taylor (1997b). The structure is a Lamé cylinder loaded by external pressure varying as $\cos 2 \Theta$. The shades of grey superposed over the regions of the structure in this figure represent the relative value of the trace measure of the modulus tensor. To mention the counterpart in the generalized cost setting to the results for this special case, note that in the former one expects a set of such shades of grey diagrams, each associated with an element of the set of design variables $B_{\gamma}$.
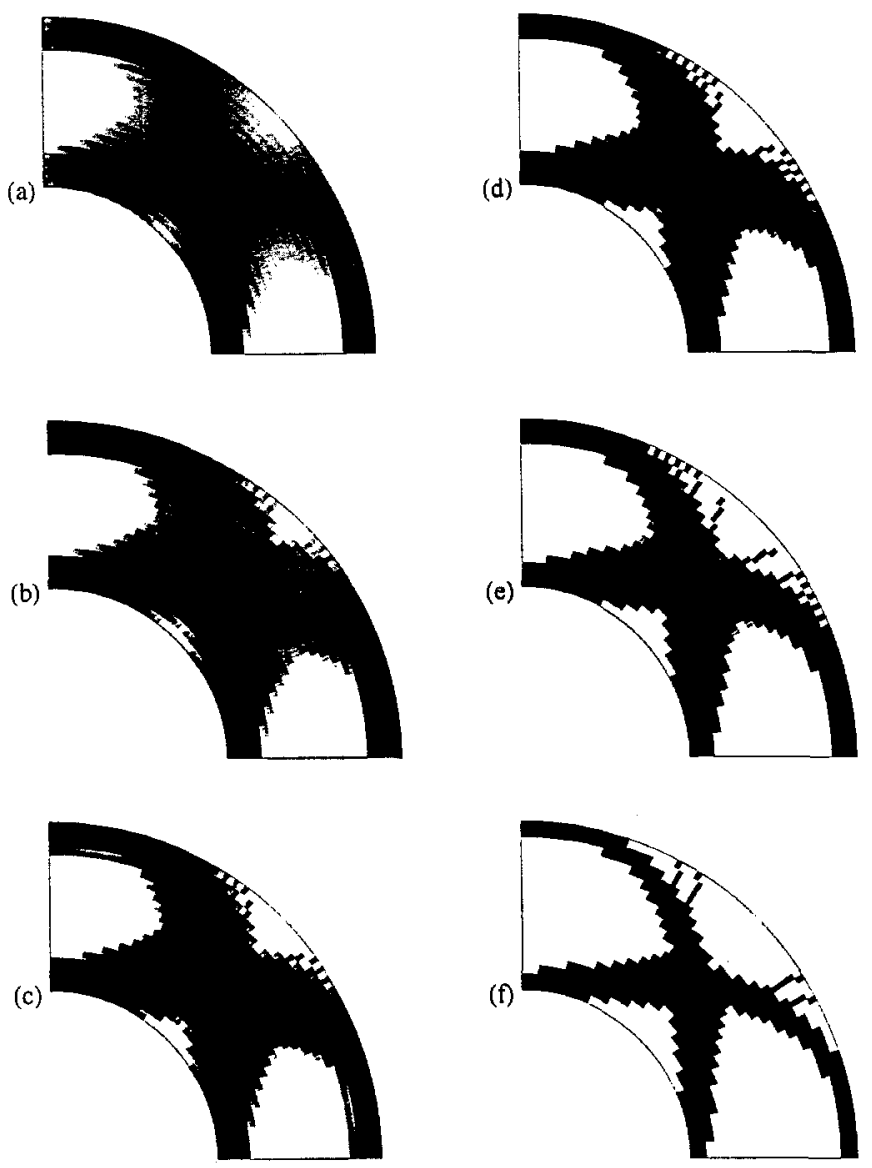

Fig. 1. Optimal designs for a cylinder subject to external pressure $p(\Theta)=a \cos 2 \Theta$. Relative magnitude of the trace measure of the material modulus shown in (a) as shades of grey. Evolution toward a black/white design is pictured in (b) through (e). Final black/white topology is depicted in (f) 
To facilitate description of a method for the prediction of an optimal black/white design based on the same formulation (see e.g. Taylor 1997; Guedes and Taylor 1997a,b), the global cost constraint is restated from (25) with unit relative cost coefficient (weighting) $b(x)$ recovered

$$
\int_{\Omega} b(x) E_{i j i j} \mathrm{~d} V-R \leq 0 .
$$

The procedure for this method has a sequence of solutions to the design problem performed, where for each solution step $b(x)$ is modified as follows. A user-determined value of the measure of the trace corresponding to a relatively light grey shade is chosen, and a relatively higher value is assigned to the cost coefficient in all regions having trace value below this designated cutoff value. With such (stepwise) adjustment in the cost coefficient, the solution for the subsequent step has the trace measure of the material suppressed in those regions with higher unit cost. The limit result for a sequence of solution steps with appropriate stepwise adjustment of unit cost is an optimal design having the value of the trace measure at either its upper (black) or lower (white) value. Results for the cylinder design example are shown in Figs. 1b through e representing steps in the procedure, with Fig. If to depict the final black/white topology. In the final step of computations for this result, the trace measure is off its bound limits for only a few from among several thousand elements, and so this limit is approached effectively in the computational procedure. Also, if the value of this measure in the black regions is unity, then white areas in the sample results have value on the order of $10^{-7}$. Finally, note that in this method the optimal black/white design result is reached through a stepwise procedure where the result at each step is the solution to a (the original) convex optimal design problem.

\subsection{Identification of "local cost" to produce specific local at-} tributes

We follow the presentation of Love (1926, pp. 158-160) for a classification scheme by which groups having specific crystalline symmetries are identified acccording to their strain energy functions. By direct translation of Love's description into the present notation, reduction from the general expression with 21 terms to the form for the "monoclinic or oblique" group having 13 terms to express strain energy is obtained if the terms identified with components $E_{1123}, E_{1113}, E_{2223}$, $E_{2213}, E_{3323}, E_{3313}, E_{1223}, E_{1312}$ in the general expression are set equal to zero. Accordingly, making use of the listing (A.12) of Appendix I it may be verified that for the monoclinic group the basis elements $B_{11}, B_{12}, B_{14}, B_{15}, B_{17}$, $B_{18}, B_{19}, B_{20}$ are to be eliminated from the expressions for cost and for strain energy. As an alternative approach, design simulating one with the designated local symmetries may be induced through an optimization procedure where the restrictions on elements of the modulus tensor are enforced via appropriate treatment of the cost constraint. Specifically, if total cost is expressed in the form

$$
\sum_{k=1}^{21} b_{k} B_{k}+b_{I}\left(B_{11}-B_{1}-B_{5}\right)+
$$

$(k \neq 11,12$,

$14,15,17$,

$18,19,20)$

$b_{I I}\left(B_{12}-B_{1}-B_{6}\right)+b_{I I I}\left(B_{14}-B_{2}-B_{5}\right)+$

$b_{I V}\left(B_{15}-B_{2}-B_{6}\right)+b_{V}\left(B_{17}-B_{3}-B_{5}\right)+$

$b_{V I}\left(B_{18}-B_{3}-B_{6}\right)+b_{V I I}\left(B_{19}-B_{4}-B_{5}\right)+$

$b_{V I I I}\left(B_{20}-B_{4}-B_{6}\right)$,

then the design produced when all coefficients $b_{I}, \ldots, b_{V I I I}$ have value large relative to the remaining coefficients $b_{k}$ is a simulation of the monoclinic local structure. Toward an understanding of why the simulation is produced, we note that the technique here is similar to the one for the prediction of optimal black/white design described in the section just above. As in that application, the desired symmetry forms in fact are identified with a limit as the $b_{I}, \ldots, b_{V I I I}$ grow large without bound relative to $\left|b_{k}\right|$, with control so that the net value of the associated terms in (27) approach zero.

To summarize, for the case described the material properties in an optimal design produced with total cost expressed in terms of the reduced basis generally belongs to the classification group "monoclinic". The particular form within that group depends on the values prescribed for the unit cost coefficients of basis terms remaining in the measure of cost. Of course, materials with lower order symmetries may result as well. The alternative approach to identify with a symmetry group may be useful in the construction of a general purpose program, one where the option exists to specify the symmetry group within the most general model of material via specification of the unit relative cost coefficients.

The next more restrictive symmetry group is the rhombic, for which there are just nine independent terms in its strain energy. If the same approach would be applied to restrict material properties in the optimal design result to those belonging to this group, then the additional basis terms $B_{10}, B_{13}, B_{16}$ and $B_{21}$ are to be deleted from the problern. Again, the reduction accomplished in this way may be verified to represent the rhombic symmetry group through a direct translation of notation from Love's to the present form. Here as in the monoclinic example, either the direct approach or the option that leads to the desired result by an inductive procedure may be applied to produce designs having material properties within this group. The actual procedures for producing the reduced model associated with "rhombic local properties" is the same as that already described, so no further consideration is given here to this example.

Six additional symmetry groups are given in Love's presentation, each group providing a variety of classes of material properties, so that altogether 27 specific symmetry structures are listed there. A number of other specific, characteristic material property structures also are identified in this section of Love's book (including a material with negative Poisson's 
ratio!), and the reader is referred to this rich resource and to the contemporary literature [e.g. the introductory text on materials by Callister (1994)] as well for additional consideration of special materials.

\section{Conclusions}

With the interpretation of both generalized cost for the optimal design problem and the mechanical strain energy in terms of a common basis, optimality conditions for the general design of continuum structures take on an especially simple and revealing form. This result, which states that in regions of the continuum where the design is free of local constraints, the optimal material is (the) one for which all components of unit strain energy are proportional to the relative unit cost of like components in the isoperimetric cost constraint, is itself an extension of familiar requirements in earlier experience with less general representations for cost. The parallelism between unit cost and unit energy follows from the use of a common basis, but the property holds independent of the specific choice among possible bases. One may appreciate in this way that the problem formulation provides a measure of flexibility, and that the freedom to select reference strains at will can be advantageous, e.g. when certain directional attributes of the material (modulus tensor) are to be introduced as data in the design problem.

Noting that the set of admissible modulus tensors in the design problem covers all possible materials within the context of classical elasticity theory, and that the expression for cost spans the space of such materials, it may be judged that - depending on the particular form of the measure of cost - material properties predicted as a part of an optimal design may be those corresponding to any modulus tensor from among those in the admissible set. In other words, any one among possible materials may turn up as the (pointwise) optimal material. This property suggests consideration of the following inverse problem, namely, given an arbitrary material, does there exist an expression of unit cost such that the given material is optimal? Restricting attention again to regions free of local constraints on design, the answer is affirmative. Given linearity in the relation between unit strain energy and unit cost coefficients, this conclusion is supported simply on the basis of the completeness of the set of admissible modulus tensors in the formulation.

While in the generalized cost formulation the basis components appear as independent elements of design, the structural material composed of them has effective properties as would be predicted for a mixture. Viewing this mixture as a composite material, a variety of interesting interpretations for application of the model are possible. As was noted in the applications section, using the procedure described there an optimal topology may be predicted for any one or more of the basis components. In the $2 \mathrm{D}$ case, for example, the resulting optimal structural material may be viewed as a layered composite having discrete components, each identified with and corresponding layer-by-layer to the properties of the topology design for the basis components. Likewise, an interpretation for $3 \mathrm{D}$ structures would be possible if one allows that where the components overlap the resulting optimal material is comprised (still!) of a mixture of the intersecting components [this is reminiscent of other design situations requiring an interpretation for "overlapping materials"; see e.g. Rozvany et al. (1982)]. Noting at the same time that any one or more of the basis elements may be held fixed while the remaining ones are to be designed, it becomes clear that the general model offers a broad range of possible interpretations.

\section{Acknowledgements}

The author is grateful to his colleague Pete Washabaugh for ongoing inspired and fruitful collaboration, in the present setting especially for the cooperation dating from 1994 that led to the original developments on generalized cost. The present work also benefited more recently from constructive discussions with Kuba Pawlicki, Helder Rodrigues, Ciro Soto, and Sergio Turteltaub. Finally, the research effort leading to the results reported here was facilitated in part using much appreciated funds received from the Ford Motor Company under Project \#95-106R.

\section{References}

Achtziger, W.; Bendsøe, M.P.; Taylor, J.E. 1997: Bounds on the effect of progressive structural degradation. J. Mech. \& Phys. Solids (to appear)

Bendsøe, M.P. 1995: Optimization of structural topology, shape, and material. Berlin, Heidelberg, New York: Springer

Bendsøe, M.P.; Mota Soares, C.A. (eds.) 1993: Topology design of structures. Dordrecht: Kluwer

Bendsøe, M.P.; Diaz, A.; Lipton, R.; Taylor, J.E. 1995: Optimal design of material properties and material distribution for multiple loading conditions. Int. J. Num. Meth. Engrg. 38, 1149-1170

Bendsøe, M.P.; Guedes, J.M.; Haber, R.B.; Pedersen, P.; Taylor, J.E. 1994: An analytical model to predict optimal material properties in the context of optimal structural design. J. Appl. Mech. 61, 930-937

Bendsøe, M.P.; Guedes, J.M.; Plaxton, S.; Taylor, J.E. 1996: Optimization of structure and material properties for solids composed of softening material. Int. J. Solids \& Struct. 33, 1799-1813

Callister, W.D., Jr. 1994: Materials science and engineering An introduction (3-rd edition). New York: John Wiley \& Sons

Diaz, A.; Lipton, R.; Ciro A.S. 1995: A new formulation of the problem of optimum reinforcement of Reissner-Mindlin Plates. Comp. Meth. Appl. Mech. Engrg. 123, 121-139

Guedes, J.M.; Taylor, J.E. 1997a: On the prediction of material properties and topology for optimal continuum structures. Struct. Optim. 14, 193-199

Guedes, J.M.; Taylor, J.E. 1997b: An alternative approach for the prediction of optimal structural topology Proc. McNU 97 Joint ASME/ASCE/SES Summer Meeting (held at Northwestern University), (to appear)

Jemiolo, S.; Telega J.J. 1997: Representations of tensor functions and applications in continuum mechanics. Report of IPPT-PAN. Warsaw: ATOS Poligrafia-Reklama 
Lipton, R. 1994: Composites with symmetry and their extremal properties. Int. J. Solids \& Struct. 31, 3407-3417

Love, A.E.H. 1926: A treatise on the mathematical theory of elasticity (4-th edition). New York: Dover Publications

Olhoff, N.; Jacobsen, J.B.; Ronholt, E. 1997: Three-dimensional structural topology and layout optimization based on optimum microstructures. Extended Abstracts of WCSMO-2, Second World Congress on Structural and Multidisciplinary Optimization (held in Zakopane, Poland)

Pedersen, P. 1993: Optimal orientation of anisotropic materials/optimal distribution of anisotropic materials, optimal shape design with anisotropic materials In: Rozvany, G.I.N. (ed.): $O p$ timization of large structural systems (Proc. NATO/DFG ASI, held in Berchtesgaden, Germany, 1991), pp. 649-682. Dordrecht: Kluwer

Rozvany, G.I.N. 1976: Optimal design of flexural systems. Oxford: Pergamon

Rozvany, G.I.N.; Bendsøe, M.P., Kirsch, U. 1995: Layout optimization of structures. Appl. Mech. Rev. 48, 41-119

Rozvany, G.I.N.; Olhoff, N.; Cheng, G.; Taylor, J.E. 1982: On the solid plate paradox in structural optimization. J. Struct. Mech. 10, $1-32$

Taylor, J.E.; Washabaugh P.D. 1995a: A generalized expression of cost for prediction of the optimal material properties tensor. In: Monteiro Marques, M.D.P.; Rodrigues, J.F. (eds.) Trends in application of mathematics to mechanics. Essex: Longman

Taylor, J.E.; Washabaugh, P.D. 1995b: A generalized expression of cost for prediction of the optimal material properties tensor. Proc. Summaries, ASME Summer Mechanics Meeting (held at UCLA)

Taylor, J.E.; Washabaugh P.D. 1997: On structural optimization formulations with generalized cost constraints. In: Godoy, L.; Rysz, M.; Suarez, L. (eds.) Proc. PACAMV (held in San Juan, Puerto Rico)

Taylor, J.E. 1997: On the prediction of topology and local properties for optimal truss design. Struct. Optim. 14, 53-62

Washizu, K. 1982: Variational methods in elasticity and plasticity. Oxford: Pergamon

Zheng, Q.S. 1994: Theory of representation for tensor functions - A unified invariant approach to constitutive equations. Appl. Mech. Rev. 47, 545-587

Appendix I. The energetic basis for a specific designated set of "reference strains"

First, the complete set of calculations for the expression of an energy basis is exemplified in the following for the 2D case [this material amounts to an elaboration on what is given by Taylor and Washabaugh (1995a)]. As the first step in this process, reference strains $\eta_{i j}^{\gamma} ; \gamma=1,2,3$ are designated

$\eta_{i j}^{\gamma}=\left\{\left[\begin{array}{ll}1 & 0 \\ 0 & 0\end{array}\right] ;\left[\begin{array}{ll}0 & 1 / \sqrt{2} \\ 1 / \sqrt{2} & 0\end{array}\right] ;\left[\begin{array}{ll}0 & 0 \\ 0 & 1\end{array}\right]\right\}$

and for the associated independent linear combinations $\zeta_{i j}^{\mu}$; $\mu=1,2,3$ one may find

$\zeta_{i j}^{\mu}=\left\{\frac{1}{\sqrt{2}}\left(\eta^{1}+\eta^{3}\right) ; \frac{1}{\sqrt{2}}\left(\eta^{1}+\eta^{2}\right) ; \frac{1}{\sqrt{2}}\left(\eta^{3}+\eta^{2}\right)\right\}=$ $\left\{\left[\begin{array}{ll}1 \sqrt{2} & 0 \\ 0 & 1 \sqrt{2}\end{array}\right] ;\left[\begin{array}{ll}1 \sqrt{2} & 1 / 2 \\ 1 / 2 & 0\end{array}\right] ;\right.$

$\left.\left[\begin{array}{ll}0 & 1 / 2 \\ 1 / 2 & 1 / \sqrt{2}\end{array}\right]\right\}$

The corresponding basis energies (no sum on $\gamma, \mu$ )

$B_{k}=\left\{\frac{1}{2} E_{i j k \ell} \eta_{i j}^{\gamma} \eta_{k \ell}^{\gamma} ; \frac{1}{2} E_{i j k \ell} \zeta_{i j}^{\mu} \zeta_{k \ell}^{\mu}\right\}$,

are evaluated as

$B_{k}=\left\{E_{1111} / 2 ; E_{1212} ; E_{2222} / 2 ;\right.$

$\left(E_{1111}+2 E_{1122}+E_{2222}\right) / 4$

$\left(E_{1111}+2^{3 / 2} E_{1112}+2 E_{1212}\right) / 4$;

$\left.\left(E_{2222}+2^{3 / 2} E_{1222}+2 E_{1212}\right) / 4\right\}$.

The inverse relation is given by

$E_{1111}=2 B_{1}, \quad E_{1212}=B_{2}, \quad E_{2222}=2 B_{3}$,

$E_{1222}=\left(-B_{2}-B_{3}+2 B_{6}\right) / \sqrt{2}$,

$E_{1112}=\left(-B_{1}-B_{2}+2 B_{5}\right) / \sqrt{2}$,

$E_{1122}=-B_{1}-B_{3}+2 B_{4}$.

Substitution of the chosen reference strains into (6) leads to the expression for strain components $\varepsilon_{i j}$ as

$\varepsilon_{11}=c_{1}, \quad \varepsilon_{22}=c_{3}, \quad \varepsilon_{12}=c_{2} / \sqrt{2}$.

Both algebraic and numerical results for the unit strain energy, say $U$, also are demonstrated in this Appendix. The expression from (14) for strain energy is recalled

$U:=\frac{1}{2} E_{i j k \ell} \varepsilon_{i j} \varepsilon_{k \ell}=$

$\frac{1}{2} E_{i j k \ell}\left(\sum_{\alpha=1}^{N} c_{\alpha} \eta_{i j}^{\alpha}\right)\left(\sum_{\beta=1}^{N} c_{\beta} \eta_{i j}^{\beta}\right)$.

The latter product is written in expanded form as (for the 2D case, still)

$U=\frac{1}{2} E_{i j k \ell} \sum_{r=1}^{3}\left(c_{r}\right)^{2} \eta_{i j}^{r} \eta_{k \ell}^{r}+$

$E_{i j k \ell} \sum_{r=1}^{2} \sum_{s=1}^{3-r} c_{r} c_{r+s} \eta_{i j}^{r} \eta_{k \ell}^{r+s}=$

$\frac{1}{2} E_{i j k \ell}\left[\left(c_{1}\right)^{2} \eta_{i j}^{1} \eta_{k \ell}^{1}+\left(c_{2}\right)^{2} \eta_{i j}^{2} \eta_{k \ell}^{2}+\left(c_{3}\right)^{2} \eta_{i j}^{3} \eta_{k \ell}^{3}\right]+$ 
$E_{i j k \ell}\left[c_{1} c_{2} \eta_{i j}^{1} \eta_{k \ell}^{2}+c_{1} c_{3} \eta_{i j}^{1} \eta_{k \ell}^{3}+c_{2} c_{3} \eta_{i j}^{2} \eta_{k \ell}^{3}\right]$

With the substitution of the designated values for reference strain, this expression is reduced to the simplified result

$U=\frac{\left(c_{1}\right)^{2}}{2} E_{1111}+\left(c_{2}\right)^{2} E_{1212}+\frac{\left(c_{3}\right)^{2}}{2} E_{2222}+$

$\sqrt{2} c_{1} c_{2} E_{1112}+c_{1} c_{3} E_{1122}+\sqrt{2} c_{2} c_{3} E_{1222}$.

Comparing this result to the latter part of (14) [i.e. the expression $\sum_{\delta=1}^{N+C} e_{\delta} B_{\delta}$ ], and recalling the earlier evaluation of $B_{k}$, the coefficients $e_{k}$ are determined directly as

$e_{1}=\left(c_{1}\right)^{2}-c_{1} c_{2}-c_{1} c_{3}, \quad e_{2}=\left(c_{2}\right)^{2}-c_{2} c_{1}-c_{2} c_{3}$,

$e_{3}=\left(c_{3}\right)^{2}-c_{3} c_{1}-c_{3} c_{2}, \quad e_{4}=2 c_{1} c_{3}$,

$e_{5}=2 c_{1} c_{2}, \quad e_{6}=2 c_{2} c_{3}$.

This completes the evaluation of all expressions related to the basis and expansions for the 2D problem.

Comparable results in $3 \mathrm{D}$ (or higher dimension!) are obtained by application of the formulae given in the text. The information is needed for the interpretation of crystal moduli given in the section on applications. To consider this case, suppose the six independent components of reference strain eta for the symmetric two-tensor in 3D are designated as

$$
\begin{aligned}
& \eta_{i j}^{1, \ldots, 6}=\left[\begin{array}{lll}
1 & 0 & 0 \\
0 & 0 & 0 \\
0 & 0 & 0
\end{array}\right] ;\left[\begin{array}{lll}
0 & 0 & 0 \\
0 & 1 & 0 \\
0 & 0 & 0
\end{array}\right] ;\left[\begin{array}{lll}
0 & 0 & 0 \\
0 & 0 & 0 \\
0 & 0 & 1
\end{array}\right] ; \\
& {\left[\begin{array}{lll}
0 & 1 & 0 \\
1 & 0 & 0 \\
0 & 0 & 0
\end{array}\right] ;\left[\begin{array}{lll}
0 & 0 & 0 \\
0 & 0 & 1 \\
0 & 1 & 0
\end{array}\right] ;\left[\begin{array}{lll}
0 & 0 & 1 \\
0 & 0 & 0 \\
1 & 0 & 0
\end{array}\right] .}
\end{aligned}
$$

The six squared terms for these eta plus fifteen independent cross-product terms, calculated as indicated for the $2 \mathrm{D}$ example, comprise the requisite set of 21 basis strain energies. For indices $\alpha, \beta, \mu$ as defined in (9), an example set of index values for the fifteen cross-product terms is

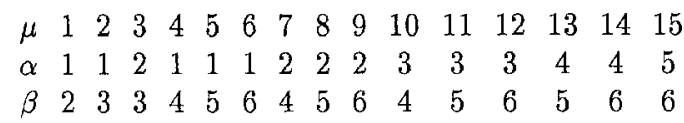

The calculations required to evaluate the basis energies are routine, following the pattern described for the $2 \mathrm{D}$ case. Results for the $3 \mathrm{D}$ counterpart of (A5), i.e. components of the modulus tensor in terms of the basis energies, are (these expressions were generated by Pete Washabaugh using Mathematica)

$$
\begin{aligned}
& E_{1111}=2 B_{1}, \quad E_{2222}=2 B_{2}, \quad E_{3333}=2 B_{3}, \\
& E_{1112}=\left(-B_{1}-B_{4}+B_{10}\right) / 2, \\
& E_{1113}=\left(-B_{1}-B_{6}+B_{12}\right) / 2,
\end{aligned}
$$

$$
\begin{aligned}
& E_{1212}=B_{4} / 2, \quad E_{1122}=\left(-B_{1}-B_{2}+B_{7}\right), \\
& E_{1313}=B_{6} / 2, \quad E_{1133}=\left(-B_{1}-B_{3}+B_{8}\right), \\
& E_{1333}=\left(-B_{3}-B_{6}+B_{18}\right) / 2, \\
& E_{1233}=\left(-B_{3}-B_{4}+B_{16}\right) / 2, \\
& E_{2221}=\left(-B_{2}-B_{4}+B_{13}\right) / 2, \\
& E_{2223}=\left(-B_{2}-B_{5}+B_{14}\right) / 2, \\
& E_{2233}=\left(-B_{2}-B_{3}+B_{9}\right), \\
& E_{2333}=\left(-B_{3}-B_{5}+B_{17}\right) / 2, \\
& E_{3132}=\left(-B_{5}-B_{6}+B_{21}\right) / 4, \\
& E_{1123}=\left(-B_{1}-B_{5}+B_{11}\right) / 2, \\
& E_{3221}=\left(-B_{4}-B_{5}+B_{19}\right) / 4, \\
& E_{1322}=\left(-B_{2}-B_{6}+B_{15}\right) / 2, \\
& E_{1213}=\left(-B_{4}-B_{6}+B_{20}\right) / 4, \quad E_{2323}=B_{5} / 2 .
\end{aligned}
$$

The expression for strain energy may be obtained from the expansion of (A9) applied with these results and the assumed set $\eta_{i j}^{1, \ldots, 6}$ of $(\mathrm{A} 11)$

\section{Appendix II. Relationship between "max $\int_{\Omega} b_{k} B_{k} \mathrm{~d} V$ " and "minimum compliance"}

Recall that solution $\ddot{u}$ to (1) equilibrates the specified loads, while $\dot{u}=\Lambda \ddot{u}$ represents the solution to the inner (minimization) problem of [D1]. As was noted in the section on "elastostatics restated in isoperimetric form", $\dot{u}$ satisfies

$$
\int_{\Omega} f_{i} \dot{u}_{i} \mathrm{~d} V+\int_{\Gamma_{t}} t_{i} \dot{u}_{i} \mathrm{~d} S=\underline{W} .
$$

The result of integration by parts of the product of $\dot{u}$ and (2) can be stated as

$\int_{\Omega} E_{i j k \ell} \dot{u}_{i, j} \dot{u}_{k, \ell} \mathrm{d} V=\dot{\Lambda}\left[\int_{\Omega} f_{i} \dot{u}_{i} \mathrm{~d} V+\int_{\Gamma_{t}} t_{i} \dot{u}_{i} \mathrm{~d} S\right]$.

Replacing the left side by the measure of "strain energy" of deformation $\dot{u}$ in terms of $B_{k}$, and with the substitution from (A13) on the R.S., (A14) becomes

$2 \int_{\Omega} e_{k} B_{k} \mathrm{~d} V=\dot{i} \underline{W}$. 
For convenience, (4) is restated here

$$
\dot{A}=\frac{\underline{W}}{\left(\int_{\Omega} f_{i} \ddot{u}_{i} \mathrm{~d} V+\int_{\Gamma_{t}} t_{i} \ddot{u}_{i} \mathrm{~d} S\right)} .
$$

Finally, by elimination of $\dot{A}$ between (A15) and (4), one may find

Received Oct. 20, 1997

Revised manuscript received March 9, 1998
$\int_{\Omega} f_{i} \ddot{u}_{i} \mathrm{~d} V+\int_{\Gamma_{t}} t_{i} \ddot{u}_{i} \mathrm{~d} S=\frac{\underline{W}^{2}}{2 \int_{\Omega} \dot{e}_{k} B_{k} \mathrm{~d} V}$.

The L.S. of (A16) measures compliance under the specified loads, while the denominator on the R.S. equals twice the argument of "max" in [D1] [recall that (A16) holds for arbitrary structure]. Thus maximization on this argument corresponds to minimization of compliance, and so the form of the problem represented in [D1] is justified. Noting again that the problem described in [D1] is a maxmin problem, the system of ${ }^{*}$ conditions for stationarity w.r.t. variation in design is sufficient to identify the optimum structure. 